\title{
SIMULAÇÃO DEM DO ÂNGULO DE REPOUSO DINÂMICO DE PARTÍCULAS DE POLIETILENO
}

\author{
N. P. ALMEIDA ${ }^{1}$, K.G. SANTOS ${ }^{1}$ \\ ${ }^{1}$ Universidade Federal do Triângulo Mineiro, Departamento de Engenharia Química \\ E-mail para contato: nataliapazin.engquim@gmail.com
}

\begin{abstract}
RESUMO - A simulação computacional é uma importante ferramenta na compreensão de processos com escoamento granular. O Método de Elementos Discretos (DEM) representa de maneira eficiente o comportamento discreto em sistemas particulados. Este trabalho estudou simulações de um tambor rotativo de bancada contendo partículas de polietileno. Foram avaliados os parâmetros coeficiente de restituição, coeficiente de fricção e coeficiente de elasticidade. A análise estatística das simulações de polietileno permitiu observar que o parâmetro do modelo mola-amortecedor que mais influencia a resposta simulada do ângulo de repouso dinâmico do polietileno é o coeficiente de atrito.
\end{abstract}

\section{INTRODUÇÃO}

A modelagem de operações com partículas é frequentemente realizada pela aproximação Euler-Euler, que é eficaz para sistemas particulados densos, com partículas esféricas e de maior densidade.

Essa abordagem, porém, não contempla as características individuais das partículas sólidas quanto à sua forma e tamanho e não identifica efetivamente a influência dessas características na fluidodinâmica da partícula (SANTOS, 2011).

O Método de Elementos Discretos (DEM) possibilita uma modelagem aprofundada da colisão partícula-partícula e partícula-parede e, dessa forma, apresenta-se como uma boa alternativa para a modelagem de sistemas particulados densos, pois contabiliza o efeito do tamanho e da forma real da partícula (GAO e SUN, 2008).

A calibração dos parâmetros de colisão de partículas é importante para o conhecimento da fluidodinâmica em diferentes equipamentos com escoamento granular, tais como, secadores rotatórios, leito fixo, leito fluidizado, leito de jorro. Os testes experimentais de ângulo de repouso estático e dinâmico das partículas são uma importante etapa desta calibração.

O ângulo de repouso dinâmico é uma característica da escoabilidade do material inclinado e o plano horizontal e caracteriza o movimento superficial do produto como se estivesse em um tambor rotativo. Este ângulo é uma função do tipo de material e suas propriedades, bem como da velocidade angular de rotação do cilindro.

Este trabalho teve como objetivo simular o ângulo de repouso dinâmico de partículas de polietileno, para posterior simulação de sua dinâmica em diferentes equipamentos. 


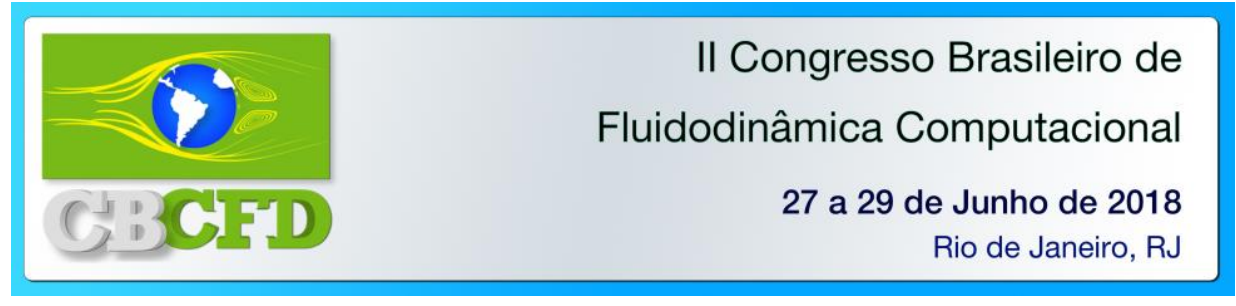

\section{MÉTODO}

A malha computacional do tambor rotatório foi gerada através do software GAMBIT®, para posterior injeção e simulação das partículas de polietileno no interior do equipamento a partir do software FLUENT®. O tambor possui $0,10 \mathrm{~m}$ de diâmetro e $0,10 \mathrm{~m}$ de comprimento, foi simulado com $50 \%$ do volume ocupado por partículas a uma rotação constante de $25 \mathrm{rpm}$.

Um planejamento composto central (PCC) foi montado, para avaliar o efeito dos parâmetros investigados: coeficiente de restituição $(\eta)$, coeficiente de fricção ou atrito $(\mu)$ e coeficiente de elasticidade $(\mathrm{k})$. As faixas dos valores de cada parâmetro investigados neste trabalho encontram-se na Tabela 1, que mostra os níveis de cada variável.

Tabela 1 - Níveis e faixas de valores das variáveis codificadas em estudo

\begin{tabular}{lllllll}
\hline & \multirow{2}{*}{ Variáveis } & \multicolumn{5}{c}{ Níveis } \\
\cline { 3 - 7 } & & $\mathbf{- 1 , 5 7 5}$ & $\mathbf{- 1}$ & $\mathbf{0}$ & $\mathbf{1}$ & $\mathbf{1 , 5 7 5}$ \\
\hline $\mathbf{X 1}$ & Coeficiente de Elasticidade [N/m] & 54,98 & 400 & 1000 & 1600 & 1945 \\
$\mathbf{X 2}$ & Coeficiente de Atrito & 0,027 & 0,2 & 0,5 & 0,8 & 0,973 \\
$\mathbf{X 3}$ & Coeficiente de Restituição & 0,027 & 0,2 & 0,5 & 0,8 & 0,973 \\
\hline
\end{tabular}

Em virtude da utilização do modelo Euleriano, o software FLUENT ANSYS® 14.0, além de resolver as equações de transporte para a fase contínua, permite simular uma fase discreta segundo uma abordagem Lagrangeana que utiliza o DEM (Discret Element Method). Esta segunda fase consiste em partículas esféricas. $\mathrm{O}$ acoplamento entre as fases e o seu impacto sobre ambas as trajetórias da fase discreta e o fluxo de fase contínua também é incluído nos cálculos. O modelo utilizado resolve para a fase fluida a Equação da Continuidade e a Equação do Movimento.

As simulações DEM foram feitas utilizando-se malhas construídas em três dimensões. O conjunto de equações de conservação e constitutivas foi resolvido utilizando-se o método dos volumes finitos. O acoplamento entre velocidade e pressão foi definido pelo algoritmo SIMPLE. Os resíduos utilizados foram menores que $10^{-3}$. Na parede foi considerada uma condição de contorno de não deslizamento. Para a discretização espacial foi utilizado o modelo QUICK e para a discretização temporal o modelo implícito de primeira ordem (SILVÉRIO, 2012).

Foi gerado um conjunto de imagens do interior do tambor em movimento para cada simulação do PCC, através das quais foi possível obter os ângulos de repouso dinâmicos médios. Em seguida, foi feita uma análise de regressão múltipla e um teste de hipóteses usando a estatística t de Student. Os coeficientes da equação estimados pelo método de mínimos quadrados com nível de significância maior que $10 \% \quad(p>0,10)$ foram negligenciados.

\section{RESULTADOS E DISCUSSÕES}

Os valores referentes às condições simuladas e as respostas para as partículas de polietileno encontram-se na Tabela 2.

Tabela 2- Ângulos médios obtidos através das simulações de partículas de polietileno 
II Congresso Brasileiro de

Fluidodinâmica Computacional

27 a 29 de Junho de 2018

Rio de Janeiro, RJ

\begin{tabular}{cccccccccc}
\hline Simulação & $\mathbf{X 1}$ & $\mathbf{X 2}$ & $\mathbf{X 3}$ & $\begin{array}{c}\text { ARD do } \\
\mathbf{P E}\left({ }^{\mathbf{0}}\right)\end{array}$ & Simulação & $\mathbf{X 1}$ & $\mathbf{X 2}$ & $\mathbf{X 3}$ & $\begin{array}{c}\text { ARD do } \\
\mathbf{P E}\left(\mathbf{(}^{\mathbf{o}}\right)\end{array}$ \\
\hline 1 & -1 & -1 & -1 & $34,36 \pm 0,99$ & 12 & 0 & 1,575 & 0 & $72,69 \pm 2,35$ \\
\hline 2 & -1 & -1 & 1 & $33,94 \pm 1,23$ & 13 & 0 & 0 & $-1,575$ & $58,25 \pm 1,25$ \\
\hline 3 & -1 & 1 & -1 & $72,85 \pm 1,44$ & 14 & 0 & 0 & 1,575 & $59,88 \pm 1,53$ \\
\hline 4 & -1 & 1 & 1 & $71,83 \pm 1,35$ & 15 & 0 & 0 & 0 & $63,61 \pm 2,70$ \\
\hline 5 & 1 & -1 & -1 & $34,24 \pm 1,14$ & 16 & 0 & 0 & 0 & $62,01 \pm 1,54$ \\
\hline 6 & 1 & -1 & 1 & $33,88 \pm 0,88$ & 17 & 0 & 0 & 0 & $63,33 \pm 1,57$ \\
\hline 7 & 1 & 1 & -1 & $63,47 \pm 2,56$ & 18 & 0 & 0 & 0 & $64,79 \pm 2,03$ \\
\hline 8 & 1 & 1 & 1 & $68,70 \pm 1,72$ & 19 & 0 & 0 & 0 & $65,00 \pm 0,92$ \\
\hline 9 & $-1,575$ & 0 & 0 & $63,45 \pm 2,41$ & 20 & 0 & 0 & 0 & $62,37 \pm 1,31$ \\
\hline 10 & 1,575 & 0 & 0 & $62,55 \pm 2,50$ & 21 & 0 & 0 & 0 & $65,34 \pm 1,71$ \\
\hline 11 & 0 & $-1,575$ & 0 & $5,20 \pm 0,10$ & & & & & \\
\hline
\end{tabular}

Na Figura 1 é possível observar os ângulos de repouso dinâmico para as partículas de polietileno, com imagens coloridas por fração volumétrica de sólidos, no tempo de $12 \mathrm{~s}$ de simulação.

1
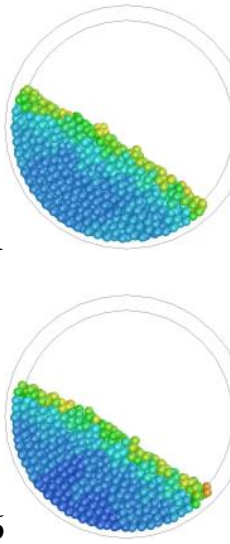

11

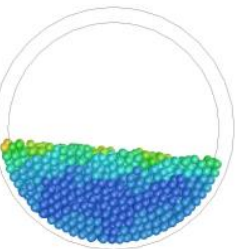

12

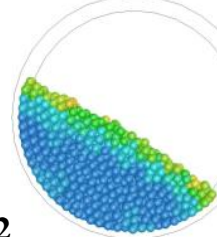

7
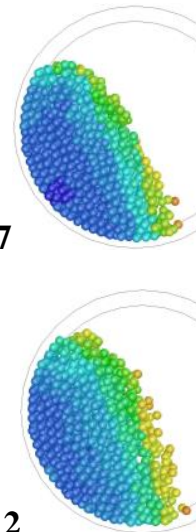

3

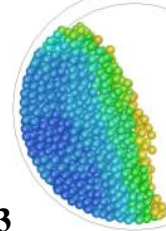

8

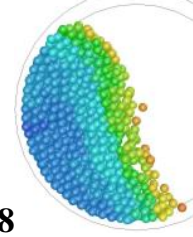

13

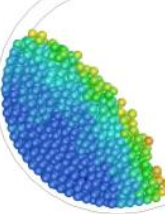

4

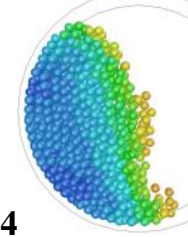

9

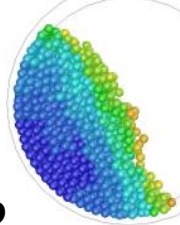

14

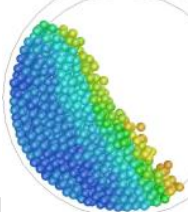

5

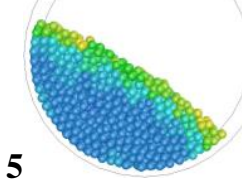

10

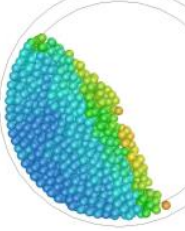

15

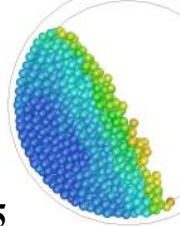

1

$3.10^{-2}$

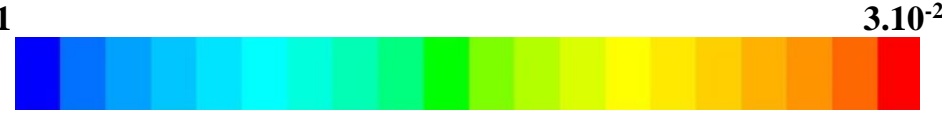

16

Figura 1 - Imagens das simulações DEM do tambor rotativo preenchido por polietileno coloridas por fração volumétrica de sólidos 


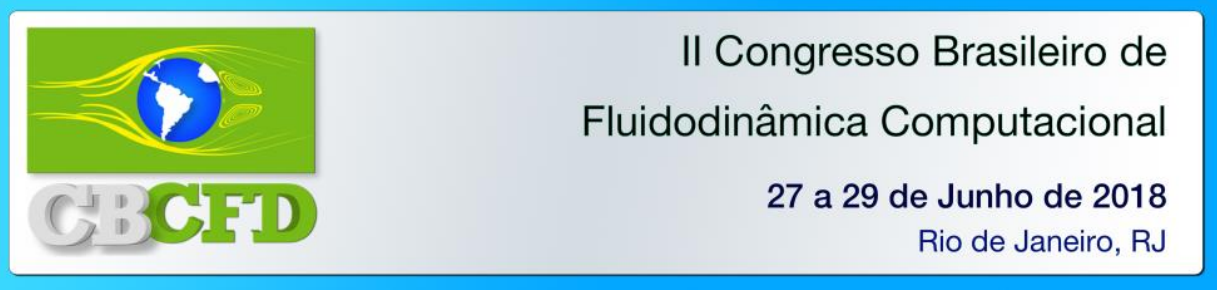

De acordo com Johnstone (2010), o ARD para pellets de polietileno varia entre $40,1^{\circ} \mathrm{e}$ 46, $3^{\circ}$. Observa-se um grande efeito da variação dos parâmetro sobre os ângulos simulados, que variaram entre $5,2^{\circ}$ e $75,9^{\circ}$. O modelo reduzido do ângulo de repouso dinâmico simulado $\left(\Phi_{P E}\right)$ é dado pela equação $1\left(\mathrm{r}^{2}=0,985\right)$.

$$
\Phi_{P E}=63,59+19,03 X_{2}-9,98 X_{2}^{2}-1,87 X_{3}^{2}-1,54 X_{1} X_{2}
$$

Em que $X_{1}$ representa o coeficiente de elasticidade $(k), X_{2}$ o coeficiente de atrito $(\mu)$ e $X_{3}$ o coeficiente de restituição $(\eta)$.

Através da análise estatística das simulações, foi possível observar que o parâmetro do modelo mola-amortecedor que mais influencia a resposta simulada do ângulo de repouso dinâmico é o coeficiente de atrito, tanto na forma linear quanto quadrática, sendo recomendado que seu valor seja medido experimentalmente, a fim de ser empregado nas simulações.

\section{CONCLUSÃO}

Para o conhecimento da fluidodinâmica das partículas, é de suma importância a calibração dos parâmetros de colisão para cada material granular.

A análise estatística das simulações de polietileno permitiu observar que o parâmetro do modelo mola-amortecedor que mais influencia a resposta simulada do ângulo de repouso dinâmico é o coeficiente de atrito, tanto na forma linear quanto quadrática, sendo que a constante da mola tem pouca influência sobre o resultado simulado quando em interação com o coeficiente de atrito.

\section{REFERÊNCIAS}

GAO, D., SUN, J. Using DEM in Particulate Flow Simulations. In Hydrodynamics Optimizing Methods and Tools, pg. 29-50, 2011.

JOHNSTONE, M.W. Calibration of DEM Models for granular materials using bulk physical tests. University of Edinburgh, 2010.

SANTOS, K. G. Aspectos fundamentais da pirólise de biomassa em leito de jorro: Cinética e fluidodinâmica do processo, FEQUI-UFU, Uberlândia - MG, 235p. Tese de doutorado, 2011.

SILVÉRIO, B. C. Estudos Fluidodinâmicos e de Secagem de Fertilizantes em Diferentes Tipos de Secadores Rotatórios, FEQUI-UFU, Uberlândia - MG. Tese de Doutorado, 2012. 